\title{
Fibre Sensing System Based on Long-Period Gratings for Monitoring Aqueous Environments
}

\author{
Catarina Silva ${ }^{1}$, João M. P. Coelho ${ }^{1}$, Paulo Caldas ${ }^{2,3,4}$ and Pedro Jorge ${ }^{3}$ \\ ${ }^{1}$ Departamento de Física, Faculdade de Ciências da Universidade de Lisboa \\ ${ }^{2}$ Departamento de Física, Faculdade de Ciências da Universidade do Porto \\ ${ }^{3}$ Unidade de Optoelectrónica e Sistemas Electrónicos, INESC Porto \\ ${ }^{4}$ Escola Superior de Tecnologia e Gestão de Viana do Castelo \\ Portugal
}

\section{Introduction}

The estuaries and coastal environments are areas of special biodiversity, being extremely sensitive to changes in its boundary conditions, particularly the properties of water, being salinity one of the most important parameters.

Thus, environmental monitoring is a crucial factor in the ecosystems health. Actually, methods for measuring salinity are mainly based on the mobility of ions in water (Zhao et al., 2003) changing the refractive index. However, these methods are inherently electrical and can be affected by any kind of electromagnetic interference. An alternative is the use of sensors based on optical technology, in particular, long-period fiber gratings (LPG) which are intrinsically sensitive to the external refractive index. These gratings devices have the advantage of being immune to electromagnetic interference and having small size, nonintrusive nature, flexible geometry, high speed of response and excellent salt water corrosion resistance.

Long-period gratings can be considered a special class of fiber Bragg gratings in which the period of the index modulation is such that it satisfies a phase matching condition between the fundamental core mode and a forward propagating cladding mode of an optical fiber. The periodic modulation of the refractive index in the fiber core typically has a period in the region from $100 \mu \mathrm{m}$ to $1000 \mu \mathrm{m}$ and a length of a few cm (James \& Tatam, 2003) which can be induced in the fiber using different process. Irradiation by ultraviolet (UV) source, electric-arc discharges, irradiation with $\mathrm{CO}_{2}$ source and application of mechanical pressure with adequate periodicity are the most common process.

The first LPG successfully inscribed in an optical fiber was described in 1996 by Vengsarkar (Vengsarkar et al., 1996a, 1996b) and was used as a band-rejection filter. In the same year Bhatia (Bhatia \& Vengsarkar, 1996) presented the first LPG device acting as a sensor. Since then, many applications for long-period gratings have been achieved in the last years and have been concentrated on the development of long-period grating-based fiber devices for use in optical communications and fiber optic sensor systems. In optical communication systems, LPGs are applied as gain equalizing filters, wavelength selective devices, band- 
pass and band rejection filters and wavelength tunable add/drop multiplexers (Vengsarkar et al., 1996a, 1996b; Dong et al., 2009). In the field of sensing systems, they can be applied as structural bend sensors, temperature sensors, axial strain sensors, refractive index sensors and biochemical optical sensors (Patrick et al., 1998a; James \& Tatam, 2002; Bhatia, 1999; Falciai et al., 2001).

The high sensitivity of LPGs to the refractive index of the surrounding environment allows them to be used as salinity sensors in aqueous media. However, in their production the removal of the buffer layer makes them a fragile structure when applied in real conditions, usually contaminated with algae and other organic materials. Taking this into consideration, this chapter addresses the potential application of long-period gratings technology in environmental monitoring. It will analyze the response of different gratings to refractive index changes, in order to evaluate and increase their sensitivity, as well as its thermal response. In this chapter, we present the issues related with LPG implementation in a real field application and review the use of LPG based sensing heads for environmental monitoring in field conditions. As a particular example, a prototype of a salinity sensor developed by the authors will be described for in situ research and capable of sustaining harsh operational conditions.

The chapter starts by presenting the main theoretical concepts related with LPGs, focusing on their fundamental aspects and fabrication methods and also its application as sensor elements (section 1). In section 2, the main configurations of refractive index sensors based on LPGs are reported. The problematic of encapsulating the sensing element and make it a robust sensor device will be addressed, in section 3. Mechanical requirements are described and the filtration issues analysed. The easiness of device cleaning is another crucial aspect that will be taken into consideration. Finally, in section 4, a prototype of a refractive index sensor for in situ research will be proposed and characterised and the potential of the sensing system as a remote refractometric device is analysed.

\subsection{Fundamentals of long period gratings}

As mentioned before, in 1996 (Vengsarkar et al., 1996a) it was reported the principle of operation of an LPG as a device based on the coupling between the fundamental mode propagating in the fiber core with the forward propagating cladding modes. This principle is illustrated in Fig. 1. The cladding modes are quickly attenuated and this results in series of loss bands in the transmission spectrum of the grating. Each of these attenuation bands corresponds to coupling to a discrete cladding mode.

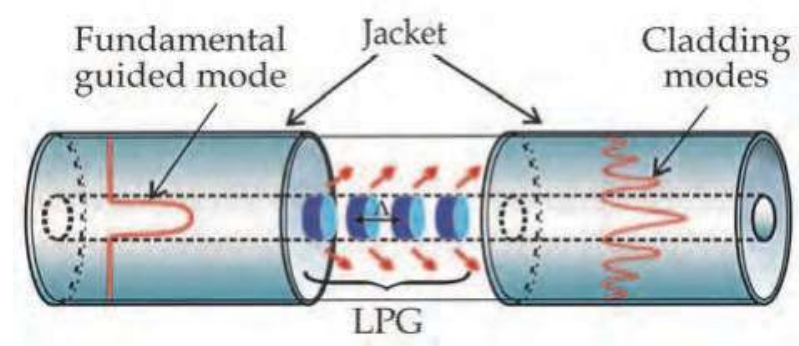

Fig. 1. Coupling of fundamental guided mode to a cladding mode in a long period grating. 
The resonant wavelength of light coupling into a particular cladding mode is given by the phase matching condition (Erdogan, 1997a, 1997b):

$$
\lambda_{\text {res }}^{m}=\left(n_{e f f, c o}-n_{e f f, c l}^{m}\right) \Lambda
$$

where $\Lambda$ is the grating period, $n_{e f f, c o}$ and $n_{e f f, c l}^{m}$ are the effective refractive indexes of the core and $m$ th-cladding modes, respectively.

The light coupled into cladding-guided modes is, most typically, absorbed in the fiber buffer or radiates away from the fiber. Thus, this type of fiber grating acts as a wavelength selective transmission filter. The wavelength transmission response of a typical LPG is shown in Fig. 2, exhibiting the characteristic attenuation bands.

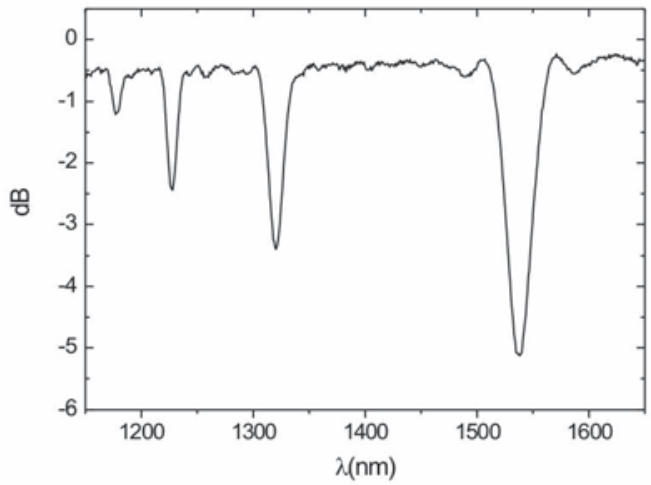

Fig. 2. Transmission response of a typical LPG.

The light transmission through the core follows a sinusoidal function of the core refractive index modulation for the wavelengths in the resonance (Zanlorensi et al., 2009)

$$
T=\cos (D L / 2)
$$

where $\mathrm{L}$ is the grating length and $\mathrm{D}$ is a coupling coefficient proportional to the core index modulation. The bandwidth of the resonance dips depends on both the coupling coefficient and the difference between the core and cladding indexes:

$$
\Delta \lambda_{F W H M}=\frac{\lambda_{m}^{2}}{\left(n_{e f f, c o}-n_{e f f, c l}^{m}\right)} \sqrt{\frac{4 D}{\pi L}}
$$

External changes in parameters like refractive index, temperature or strain can affect the terms in Eqs. (1) and (3), consequently shifting the attenuation dips and altering their bandwidths (Zanlorensi et al., 2009).

\subsection{Fabrication techniques}

LPGs are created by inducing a periodic refractive index modulation in the core of the optical fiber with period lengths of typically several hundred micrometers. This can be made by 
permanent modification of the refractive index of the optical fiber core or by the physical deformation of the fibre. Since the first demonstration of LPGs by writing the grating with UV laser light through an amplitude mask in 1996 (Vengsarkar et al., 1996a), several methods have been developed to fabricate theses structures. The most widely used method is the exposition of the photosensitive optical fibres to UV light through an amplitude mask, phase mask or by interferometry (Bhatia \& Vengsarkar, 1996; 1998a, James \& Tatam, 2002; Erdogan, 1997a). In this case, the mechanism responsible for the refractive index change is related with the formation of Germania-associated defects and the gratings can be written using light with wavelengths between 193 and 355 nm (Bhatia \& Vengsarkar, 1996; Zanlorensi et al., 2009). The amplitude mask technique consists of simply imprinting the mask pattern onto the core of a photosensitive optical fiber placed in contact with or in close proximity immediately behind the mask. The fiber exposure to the fringe pattern induces a refractive index modulation in the core with the same periodicity as that of the mask (Nikogosyan, 2006). The mask might be fabricated in chrome-plated silica or metal foil. The disadvantages of this method are that the grating period and length are restricted by the dimensions of the amplitude mask which can also be easily damaged if exposed to UV light whose intensity exceeds its damage threshold. A further useful technique was presented by Hill et al. in 1990 (Hill et al., 1990) in the form of point-by-point periodic grating manufacture. In this technique, gratings are written period after period, and a single pulse of UV light from an excimer laser passes through a mask containing a slit making the refractive index of the core in the irradiated fiber section locally increase. However, all process that depends on UV illumination requires first that the fiber must be receptive to this electromagnetic radiation. In other words, the fiber must be made photosensitive before writing the grating. This can be achieved by doping the fiber silica core with impurity atoms (such as germanium, boron) or by hydrogen loading (Kashyap et al., 2010, Lemaire et al., 1993).

Kondo et al. (Kondo et al., 1999) found that focused irradiation of femtosecond laser pulses causes a permanent refractive index increase in various glasses, and they demonstrated a novel technique for the fabrication of long-period gratings by focused irradiation of femtosecond laser pulses. They induced a refractive index periodic structure in the core of a single-mode fiber by using light that is not absorbed by the core and cladding glasses and a polymer coating. In contrast with the UV-laser induced, LPGs fabricated using infrared (IR) femtosecond lasers have high resistance to thermal decay despite having no special stabilization.

Another possibility to create these structures is by applying the ion implantation technique. This technique induces a refractive index increase in silica-based glass, which is mainly due to the densification of the silica glass induced by atomic collisions (Fujimaki et al., 2000). Disadvantages of LPG manufacture by using this technique includes the necessity of specialized equipment (such as a tandem accelerator), as well as the fact that the cladding material average refractive index is also raised in the process, which leads to large background losses in the transmission spectrum. The main advantage of ion implantation is that it is possible to inscribe LPGs in almost any type of silica-based fiber without prior photo-sensitization.

Gratings inscription was also achieved through the use of thermal effects by exposure to nearIR carbon-dioxide $\left(\mathrm{CO}_{2}\right)$ laser light (Davis et al., 1998; Kim et al., 2002) or electric arc discharges (Rego et al., 2001; Falate et al., 2006). Both techniques allow the inscription of the gratings 
without the need hydrogen-loaded or Ge-doped core fibres and are more flexible than the UV technique since it is not required the fabrication of a specific amplitude mask for each desired resonance wavelength (Humbert \& Malki, 2003). The first results of the $\mathrm{CO}_{2}$ laser application in the fabrication of LPG on fiber optics were published in 1998 (Davis et al., 1998; Akiyama et al., 1998; Liu et al., 2011). In this case, the residual stress relaxation and glass densification are usually considered to be the main mechanisms responsible by the formation of LPGs and have demonstrated very high thermal stability. In this technique, the $10.6 \mu \mathrm{m}$ wavelength laser radiation is focused to a spot by means of spherical lenses and LPGs are written by periodical local heating. The grating period is determined through the movement of a computer controlled translation stage. During the writing process, a broadband source and an optical spectrum analyzer are used to measure the transmission spectrum.

The other process used to write LPGs by periodical local heating of the fiber is based on electric arc-discharges. In this technique the refractive index modulation results from deformation of fiber due to intense heating caused by an electrical discharge transversal to the fiber. This writing method is very flexible, easy to implement and does not require the use of special fibres or high cost equipment, such as UV or IR lasers. These features make this technique particularly suitable for the low cost fabrication of LPGs. An example of a typical electric arc-discharge setup for LPGs manufacture is schematized in Fig. 3. The fabrication process consists of positioning an uncoated fiber between the electrodes of a commercial splicing machine. A small weight is suspended in one of the fibre's extremities to keep a constant longitudinal tension. The other extremity of the fiber is mounted on a computer controlled translation stage. An electrical arc discharge is applied with the fusion splicer, using an electric current of $8.5-10.0 \mathrm{~mA}$ for $0.5-2.0 \mathrm{~s}$. A translation stage moves the fibre, after each discharge, by a distance that represents the grating period (typically 300-700 $\mu \mathrm{m})$. The refractive index change is created by the fast heating and cooling discharge cycles. Due to the asymmetric nature of electric arcs, the result of each consecutive discharge is

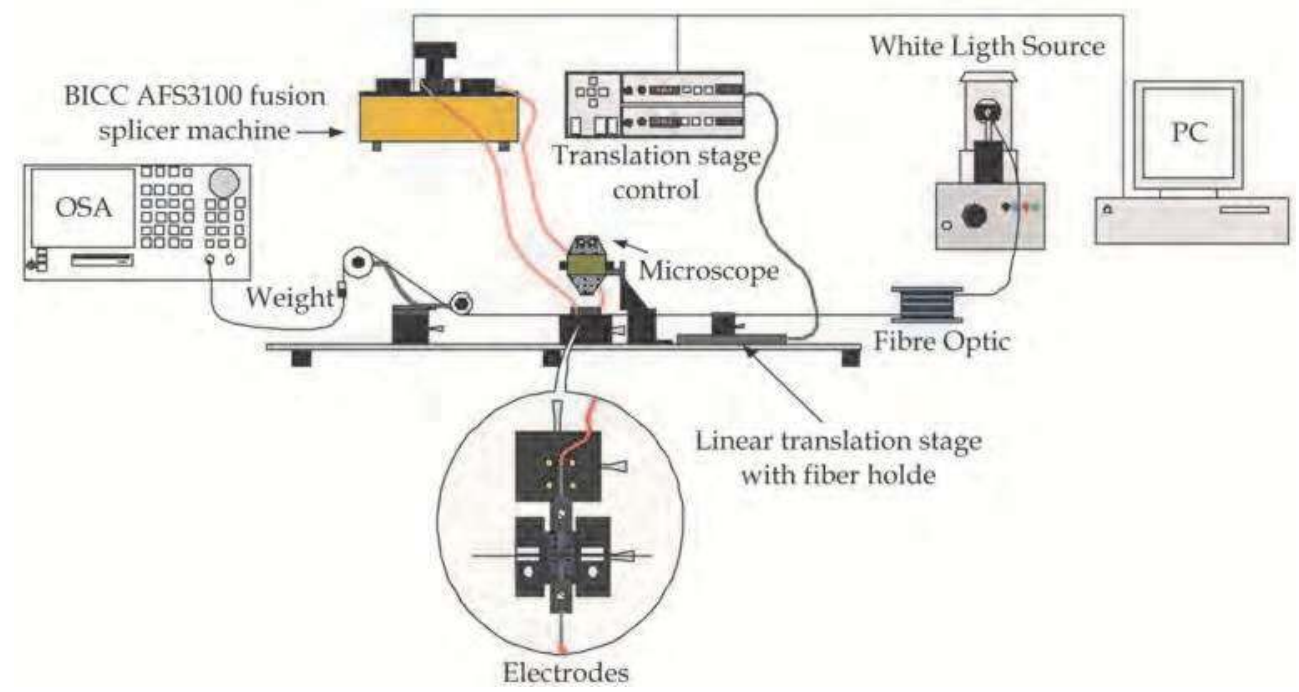

Fig. 3. Schematic diagram of LPFG fabrication system based on electric arc-discharge. 
never exactly the same. Therefore, in order to obtain an LPG with a given characteristics, the evolution of the transmitted spectrum is monitored by an optical spectrum analyser and a White Light Source (WLS). When the measured spectrum shows suitable characteristics to the intended application, the process is terminated. Usually 15-100 points are necessary to form the grating.

Since LPGs have periods of the order of hundreds of micrometers they can also be induced mechanically through periodic microbends in optical fiber (Rego et al., 2003; Yokouchi et al., 2005; Cardenas-Sevilla et al., 2009) Several configurations have been developed to produce mechanical gratings but they share the same objective, that is, to induce periodic stress and/or microbending in a single mode fibre (Rego et al., 2003). This technique, as the arc discharge technique, can potentially be applied to any kind of fibre and is also simple, flexible and low-cost. Another important advantage over other techniques is the fact that these gratings can be produced without removing the fibre coating and therefore the fibre keeps its mechanical integrity.

\subsection{Sensing characteristics}

As mentioned before, LPGs are influenced by alterations that can occur in the environment at the grating location, such as changes in the refractive index or temperature, resulting in measurable changes in the LPG transmission spectrum. This is the basis of the sensing LPGs (Bhatia, 1999). In this section, it is presented an analysis of the implementation of LPGs as an element sensor of physical parameters such as temperature, mechanical deformations and refractive index of the environment.

\subsubsection{Temperature sensitivity}

The temperature sensitivity of the LPGs may be understood by differentiating the phase matching condition equation (Humbert \& Malki, 2002) Since the contribution of the waveguide property $(d \Lambda / d T)$ to the resonance wavelength shift with temperature variation is negligible compared to that of the material property $(d n / d T)$ in LPGs the derivative of equation (1) can be set as

$$
\frac{d \lambda_{\text {res }}^{m}}{d T}=\frac{d \lambda}{d T}\left(n_{e f f, c o}-n_{e f f, c l}^{m}\right)+\Lambda\left(\frac{n_{e f f, c o}}{d T}-\frac{n_{e f f, c l}^{m}}{d T}\right) \approx \Lambda\left(\frac{n_{e f f, c o}}{d T}-\frac{n_{e f f, c l}^{m}}{d T}\right)
$$

Fig. 4 shows the wavelength response and the spectral shift of the LPG (induced in Corning SMF-28 fiber) when subjected to temperature variation from $0{ }^{\circ} \mathrm{C}$ (related to a $20^{\circ} \mathrm{C}$ ambient temperature). As expected, the resonance peaks shift to longer wavelengths with increasing temperature. The heating causes an increase in the difference between effective refractive indexes of the modes due to the thermo-optic coefficient of the core being larger than that of the cladding, causing a shift in wavelength to higher values. As a coarse approximation, a linear fit to the wavelength shift data yields a slope of $\sim 0.085 \mathrm{~nm} /{ }^{\circ} \mathrm{C}$.

\subsubsection{Refractive index measurement}

An unusual feature of LPGs is their high sensitivity to the refractive index changes in the grating location. This alteration will also change the matching condition expressed by 


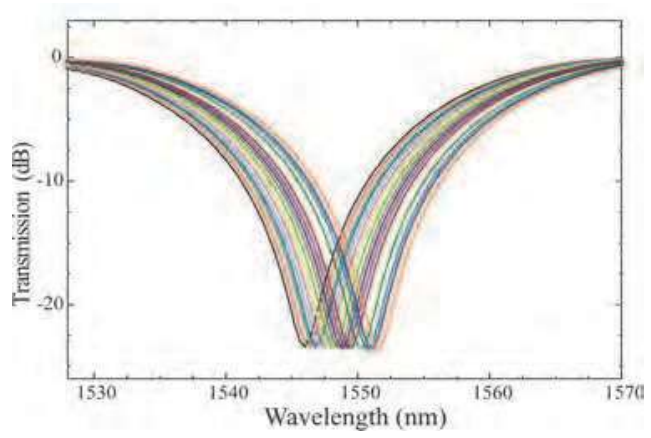

(a)

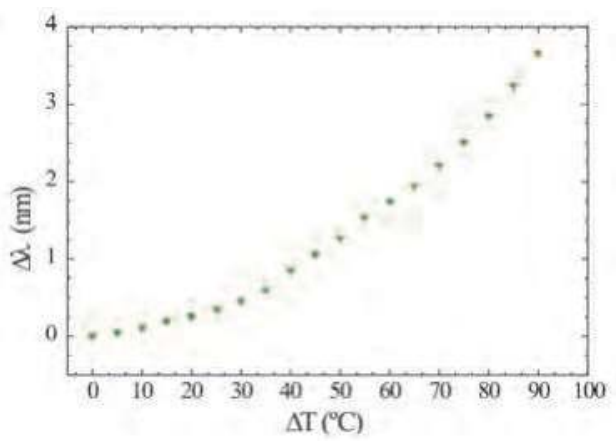

(b)

Fig. 4. (a) Transmission spectrum and (b) shift in the wavelengths of the LPG with respect to temperature variation.

equation (1) and will lead to wavelength shifts of the resonance dips in the LPG transmission spectrum. Such displacement occurs due to the dependence of the effective refractive index of the cladding modes on the refractive index of the surrounding medium. The sensibility to refractive index changes increases with the reduction of the difference between the two effective refractive indexes.

Fig.5 shows an example of the evolution of the LPG transmission spectrum when the refractive index of the external medium changes. For calibration purposes, the LPGs were immersed in liquids with different refractive indexes in the range [1.33, 1.43] at room temperature in both situations. It can be seen that as the refractive index of the external medium increases the attenuation peak shifts towards shorter wavelengths. This is an expected displacement because an increase in the effective refractive index of the cladding according to the resonance condition leads to lower resonant wavelengths (Rego et al., 2006).

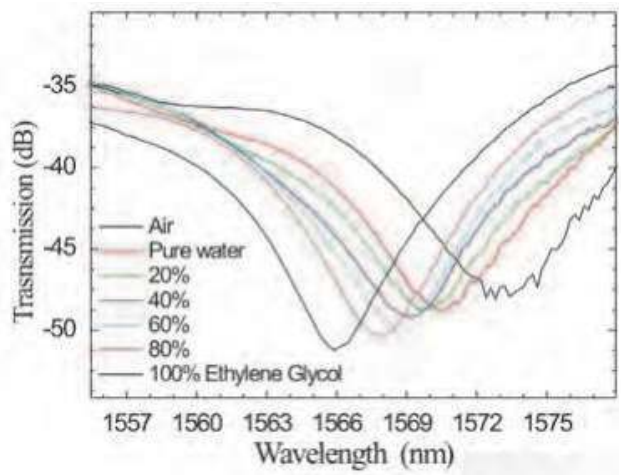

(a)

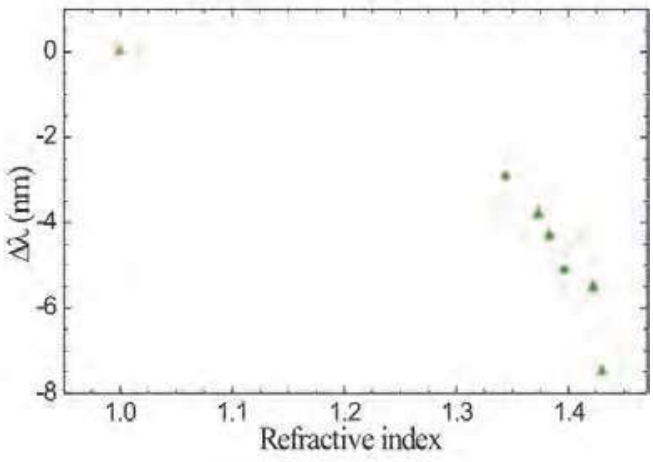

(b)

Fig. 5. (a) Transmission spectrum of long period gratings induced in Corning SMF-28, for different refractive index and (b) corresponding variation in the shift of the resonant wavelength. 
The sensitivity of LPGs to refractive index and temperature is influenced by the composition of the optical fiber. Rego (Rego et al., 2006) investigated the sensitivity of arc-induced LPGs in pure-silica-core fibres to refractive index changes of the external medium. These results show a two to three times increase of the sensitivity when compared to gratings written in standard fibres. This results from doping the cladding with fluorine which lowers its refractive index and, therefore, reduces the refractive index between the cladding and its surroundings, increasing the grating sensitivity.

As the effective refractive index of the cladding modes is strongly dependent on the cladding diameter, the easiest way to increase the sensitivity of the LPGs is by etching the fiber cladding in the region of the grating to a diameter such that the evanescent field of the mode displays a higher overlap with the immediate surrounding environment. This concept is clearly illustrated in the phase-matching condition (equation 1), where for a fixed period the change in effective refractive index for a higher order $m$ of the cladding mode results in a new resonant wavelength - the effective refractive index of the core remains unaffected. With this configuration, the value of the effective refractive index of the waveguide is directly affected by the refractive index of the medium, and this effect is stronger for higher order modes.

\section{Refractometers based on long period gratings}

In recent years the development of devices for measuring the refractive index has been the subject of great interest for applications in industrial processes and quality control, biomedical analysis, and environmental monitoring. In this context, the LPGs offer advantages, being extremely sensitive to changes in refractive index of the medium where they are immersed. However, although the use of such devices is advantageous, its use as a sensing element is limited by the relatively large rejection bandwidth $(\sim 10 \mathrm{~nm})$ which does not provide a good resolution. In this context, several schemes for refractive index sensing based on LPGs have already been proposed (James et al., 2002; Han et al., 2001).

The simple configuration, consisting of a light source, an LPG immersed in a fluid and an optical spectrum analyzer has been studied by several authors (Falciai et al., 2001; Zhu et al. 2003). Lee et al. (Lee et al., 1997) demonstrated a new analysis method to determine the spectral shift of an LPG as a function of refractive index of the medium. The change of this parameter with the wavelength of the LPG attenuation band was presented by Patrick (Patrick et al., 1998a). As previously mentioned, the sensitivity of the sensor to external refractive index change can be enhanced by decreasing its cladding diameter by chemical etching of the optical fiber (James \& Tatam, 2003). The reduction in cladding diameter changes the effective index of the cladding modes, resulting in an increase in the central wavelengths of the attenuation bands.

Gwandu et al. proposed a compact scheme for simultaneous measurements of temperature and refractive index in a medium, using two LPGs recorded in a double cladding hydrogenated fiber (Gwandu et al., 1999). The effect of the thickness of layers deposited on the grating surface regarding its response to refractive index changes has also been investigated (e.g. James et al., 2002). A thin film of organic material was deposited on an LPG through the Langmuir-Blodgett technique. The central wavelength and the minimum transmission band attenuation of the LPG depend on the thickness and refractive index 
layer of the deposited material, even when the refractive index is higher than the cladding. It was observed that for films with refractive index higher than that of the cladding, the wavelength and amplitude attenuation of the band exhibits a very high sensitivity when the film thickness is of the order of a few hundred nanometers. If the material deposited has a refractive index below that of the cladding, the sensitivity to film thickness is considerably reduced.

LPG pairs have shown high resolution to refractive index measurement compared to the use of a single LPG (Han et al., 2001; Zhang et al., 2005). The advantage of using a LPGP sensor relies upon its principle of operation, where the coupled core and cladding modes from the first LPG combine again at the second matched LPG to form interference fringes. The core and cladding paths constitute the arms of a Mach-Zehnder interferometer (MZI) in the transmission mode (Mishra et al., 2005; Murphy et al., 2007) or a Michelson interferometer in the reflection mode (Swart, 2004). A schematic diagram of these two configurations is presented in Fig. 6.

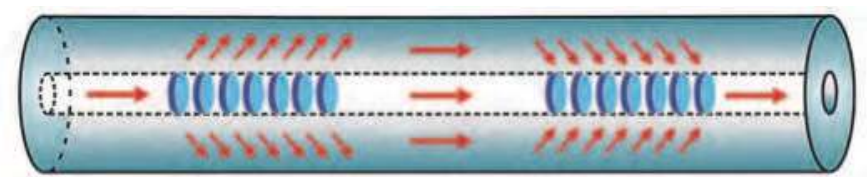

(a)

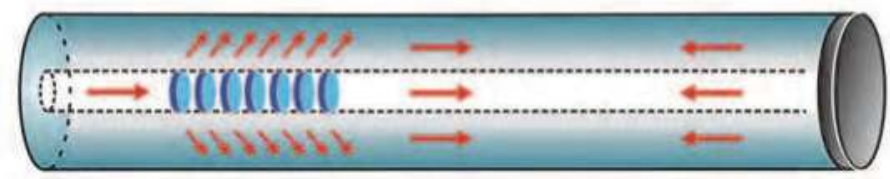

(b)

Fig. 6. Schematic diagrams of an LPG-based: (a) Mach-Zehnder interferometer; (b) Michelson interferometer.

The Mach-Zehnder interferometer operate in transmission mode and is obtained by placing in series two identical LPGs inscribed adjacent to one another such that an interferometer cavity of a certain length is formed between the two gratings. When light from a broadband source passes through the first LPG, the part that propagates in the fundamental mode is coupled to the forward propagating cladding modes. At the second LPG, the cladding mode is coupled back into the core. Due the difference between the effective refractive indexes of the core and cladding modes, the light coupled into the core by the second LPG is phase shifted with respect to the light that propagated through the core, giving rise to the interference fringes (Lee \& Nishii, 1998). The core and cladding paths constitute the arms of the Mach-Zehnder interferometer. The frequency of the fringes depends on the separation between the two gratings, while the phase is influenced by measured-induced changes in the effective index of the cladding modes (Murphy et al., 2007). The fine interference fringes in the transmission spectrum of the pair of LPGs can enhance the resolution and the sensitivity of measurement. The narrower bandwidth of the fringe facilitates greater resolution in the measurement of the wavelength than is possible with conventional LPGs (Han et al., 2001). 


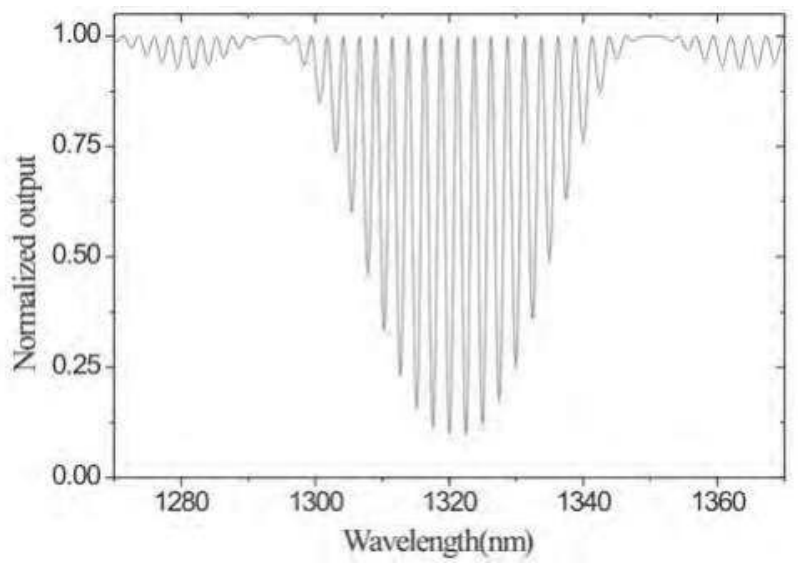

Fig. 7. Transmission spectrum of an optical fiber having two cascaded LPGs as a MachZehnder interferometer.

The in fibre-Michelson interferometer was proposed and studied by Swart (Swart, 2004). This configuration is based on a single LPG and on a fibre end mirror, as show in Fig. 6.b). In this case, the light coupled to cladding modes is reflected by the mirror and finally recoupled back to fibre core. This is a very simple structure and offers several advantages over the Mach-Zehnder configuration such as increased sensitivity, operation in reflection mode and lower manufacture complexity (it avoids the need to fabricate identical LPGs). This reflection mechanism and compact size make this kind of sensing-structure attractive as biomedical probes.

The misaligned splicing point (MSP) formed by a commercial fusion splicer may replace the first LPG in an LPG pair to create an MZI (Dong et al., 2005). In this, the coupling of light from the core to the cladding modes is made trough the MSP, as illustrated in Fig. 8.

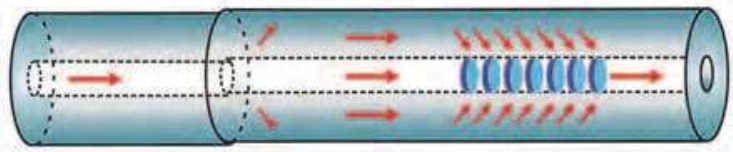

Fig. 8. Misalignment splicing point is formed to interact with the single-mode fibre-based LPG

Ding et al (Ding et al., 2005) reported the fabrication and characterization of a sensor with high sensitivity to refractive index changes based on a couple of LPGs in which it was reduced the fibre section between them (FTS-LPGP-fibre-taper seeded LPG pair). Its principle of operation is schematised in Fig. 9. This configuration allowed an increase by a factor of five in the sensitivity to refractive index change on the external environment when compared to the conventional configuration (Frazão et al., 2006).

Allsop et al. (Allsop et al., 2006), demonstrated that a Tapered-LPG configuration has a high spectral sensitivity to refractive index change in liquids. This configuration is based on the writing of a LPG centred on a taper. 


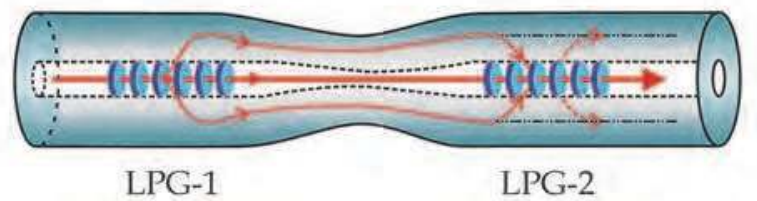

Fig. 9. Schematic diagram of a FTS-LPGP (fiber-taper seeded LPG pair).

Recent advances have been focused in the research of LPGs induced in photonic crystal fibres (PCFs) which consist of a pure-silica core, and a microstructured air-silica cladding (Yu et al., 2006). Because the microstructured cladding consists of air holes, a PCF's cladding index shows strong wavelength dependence. This makes such fibres exhibiting some properties that make them advantageous in face to conventional fibres (e.g. by reducing or even eliminating the cross-section problems).

\section{Sensing head}

Several authors have experimentally demonstrated different configurations for the application of LPGs as sensor elements. However, when the LPGs are to be deployed in harsh environments, their fragile nature makes the existing technology not satisfactory. One of the harshest environments where fiber-based sensors have a huge potential are natural aqueous media. The existence of biological activity is added to other debris that can not only alter the sensing capability but severely damage the entire sensing head.

This section addresses the several issues that must be considered in the project of a system to host a LPG in order to reduce the potential cross-sensitivity problems and obtain a high performance when applied in real conditions in natural aqueous environments. The issues related to the mechanical requirements in order to achieve a high performance sensor device under the considered conditions will be analysed. Strain constrains, supporting conditions and other operational prescriptions will be addressed. Also, the characteristics of a system developed by the authors will be described in detail.

\subsection{Mechanical requirements}

The mechanical part of the sensor head should be projected in order not only to bear the sensor element, but also give support to filtering elements (latter addressed) and allow efficient cleaning procedures, part of the unavoidable regular maintenance.

\subsubsection{Structure}

First considerations should regard the material used to produce the supporting structure. Being in direct contact with the aqueous medium this choice is of prime importance for any project. Resistance to corrosion and structural rigidity are the main characteristics to be taken in consideration, while price and machinability are also usually considered. Two main groups can be considered: metals (including alloys) and polymeric materials.

When metals and alloys interact with an environment, their behaviour is highly dependent on both the thermodynamics and kinetics of the interaction. The chemistry, solubility, 
structure, thickness, cohesion, adhesion, continuity, and mechanical properties of the reaction compounds contribute to the formation of either passivation or corrosion, two concurrent processes. In fact, passivation can be defined as the spontaneous formation of a hard non-reactive surface film inhibiting further corrosion. This layer is usually an oxide or nitride a few nanometres thick.

Contrary to metals, polymers are not affected by corrosion processes although susceptible to light (mainly, ultra violet) and potentially less structurally stable. Nevertheless, the research in engineering polymers is highly advanced and application-dedicated materials can be developed. So, depending on overall functional requirements, a wide range of materials can be chosen. The structure and components of the sensing head may use carbon steels, low alloy steels, aluminium alloys, copper and copper alloys, stainless steels, superaustenitic stainless steels, nickel-base alloys, cobalt-base alloys, ultra-high strength steels and stainless steels, titanium and titanium alloys, polymers, or composites. Newer materials such as intermetallic compounds, nano-structured alloys, and amorphous alloys are promising materials, but their collective database of respective properties is currently extremely limited (Shifler, 2005).

Choosing the correct material in the subject of this paper depends on very specific data regarding the application. If, as mentioned, the environment is a key point to consider, the kind of measurements to perform, duration of testing, production numbers (mass or small production) and available budget easily superpose. At the end, nowadays there are a sufficient number of materials that can perform well in harsh environments.

\subsubsection{Tensing element design}

Since the LPGs are intrinsically sensitive to bending curvature, which consequently influences its sensitivity to the various parameters, for field deployment in practical applications, it becomes necessary to design a support system that can avoid these cross sensitivities. A particular solution tested by the authors was a spring-based system designed in order to apply a constant force to the grating regardless of the external handling conditions. In general, the design of a compression spring must consider the following factors:

1. space for housing the spring;

2. rigidity requirements;

3. guidelines for the values of displacement and maximum force regarding the values imposed by the project.

Fig.10 shows a helical spring subjected to a compressive deformation, $y$, where $d$ is the diameter of wire and $\mathrm{p}$ the helix pitch (Shigley, 1986).

The elongation (or contraction) of a spring is determined by the torsion deformation of all the active coils of the spring. Then, the deformation of the spring coils with $\mathrm{N}_{\mathrm{a}}$ active coils under the action of a force $\mathrm{P}$ is given by

$$
y=\frac{8 P D_{m}^{3} N_{a}}{d^{4} G}
$$

being $G$ the material's elasticity modulus and $D_{m}$ the mean diameter or the coils. 


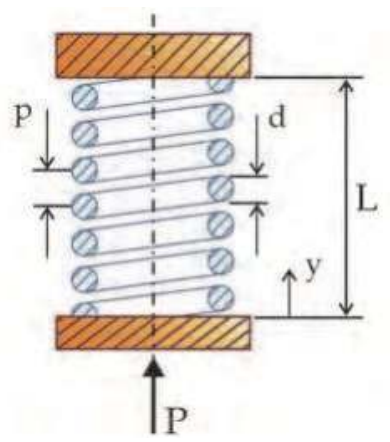

Fig. 10. Helical string subjected to compression.

The helical spring constant or stiffness, $\mathrm{K}$, which reflects the amount of deformation of a spring, $\mathrm{y}$, when subjected to a load $\mathrm{P}$, can be determined by,

$$
k=\frac{P}{y}=\frac{d^{4} G}{8 D_{m}^{3} N_{a}}
$$

Representing the length of the unloaded-spring by $\mathrm{L}_{0}$, the helix step, $\mathrm{p}$, is

$$
p=\frac{L_{0}}{N_{a}+1}
$$

and considering the spring ends as having close spirals, the total number of coils, $\mathrm{N}_{\mathrm{t}}$, is given by

$$
N_{t}=N_{a}+2
$$

\subsection{Filtering system}

Essential in the sensing head performance is the encapsulation process in view of the specifications of a filtration system. In real conditions, the system hosting the LPGs requires an adequate filtering in order to prevent the deposition of organic materials and the accumulation of particles around the sensor element, hence interfering in the measured value. The filtration process to protect the sensing head is essentially based on the passage of fluid through a porous or permeable material. The encapsulation proposed for the sensor involves two levels of filtration: one capable of retaining larger particles and another for the mud usually existing in natural aqueous environments.

For filtrating larger particles it is common the use of lower pore sizes in order to prevent the deposition of waste or algae. The filtration of mud is achieved through the process of membrane separation. This consists in running a liquid through a membrane, which acts as a selective barrier and when subjected to pressure, forced or natural, leads to the passage of fluid and smaller compounds through their pores.

The membranes can be manufactured with organic or inorganic material. Inorganic membranes exhibit higher mechanical strength, thermal and chemical robustness and have a 
longer active life. The inorganic membranes can be ceramic (zirconium oxide, aluminium or titanium), metal and graphite. The efficiency of the membrane depends on several factors, such as the properties of membrane and fluid and operating conditions (pressure, temperature, turbulence) (Queiroz et al., 2001).

Their morphology is mainly related with the density and porosity (number and dimension of the pores) of the layers constituting the membrane. The porosity, thickness, pore size and permeability are important factors in the choice of the membrane. These characteristics depend on the material of which it is made off and on the manufacturing technique. The higher the porosity the lower the resistance to the fluid flow through the membrane is. In general, the classification of the membranes is based on the structure and mechanism of separation. Regarding the structure of a membrane, it may be porous or nonporous, and symmetrical or asymmetrical, as is illustrated in Fig. 11. The anisotropic membranes are characterized by presenting a very thin, more packed, layer supported by a porous layer. In the case of porous membranes, the pore size and its distribution will determine which particles or molecules will be retained by the membrane (Queiroz et al., 2001).

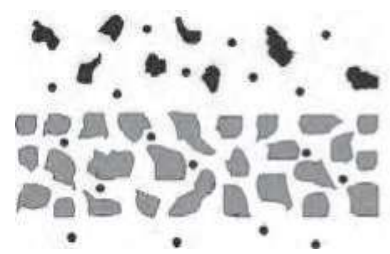

(a)

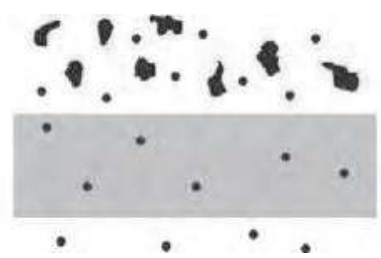

(b)

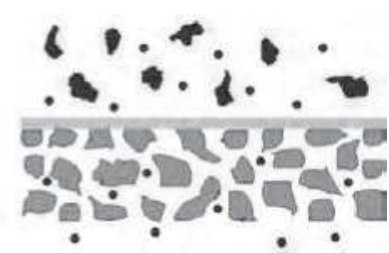

(c)

Fig. 11. Schematic representation of (a) symmetric porous and (b) non-porous membranes and (c) asymmetric porous membranes.

Among the existing membrane separation processes, we only address the micro-and ultrafiltration processes. These processes differ among themselves mainly in the pore size and pressure required for operation.

Microfiltration can be defined as a process for solid-liquid separation using membranes with pore diameters ranging from $0.1 \mu \mathrm{m}$ to $2.0 \mu \mathrm{m}$, and operating at pressures between $0.2 \mathrm{bar}$ and 2.0 bar depending on the product to be filtered. The filter is a micro-porous membrane and this technique allows you to retain suspended particles, bacteria and some ions (Queiroz et al., 2001).

The ultrafiltration process operates at pressures between 2.0 bar and 7.0 bar and uses membranes with micro-pores of $1 \mathrm{~nm}$ to $100 \mathrm{~nm}$, which allows the passage of small molecules (water, salts) retaining all suspended solids including those causing turbidity and even micro-organisms. The flow through the membrane is controlled by the pressure exerted on the liquid. The main limitation of this process is the membrane blockage, which occurs when particles are deposited on the filter inside leading to a decrease in its filtration capacity. The choice of a membrane with pores smaller than the diameter of the particle filter and subjected to a pressure gradient, prevents this phenomenon. Operating conditions such as flow, velocity and pressure concentrated in the pores are also factors that influence the degree of clogging of the membranes. The application of a pressure gradient in the membrane in contact with the solution promotes a flow of fluid through the membrane. 
The main advantages of membrane separation processes are:

1. high selectivity by using one process or the coupling of processes;

2. generally, these processes operate at room temperature, it is not necessary to change the fluid temperature to promote the phase separation;

3. low power consumption: promoting the separation that occurs without phase change;

4. easy to combine with other processes.

The main disadvantage is its high cost. However, costs associated with the application of this technology have been reduced considerably, since the membranes are produced on a larger scale, there is a greater number of firms in the market and, when properly applied, the membranes have a longer service life and flow permeated most stable and high.

\subsection{Prototype}

The guidelines explained in the previous text were applied in the design of a mechanical device with the functions of supporting and housing a sensor for measuring salinity and temperature, which is based on a LPG inscribed in standard silica optical fibre, with $125 \mu \mathrm{m}$ in diameter. The developed system is intended to operate at half depth in the Ria de Aveiro, inserted in the optical cable, protecting the optical fibre (Silva et al., 2010).

\subsubsection{Mechanical device}

Being a prototype and not expected to operate for longer periods of time the main driver for the choice of the material was cost an easy of manufacture. For this reason, common aluminium (1060 Alloy) was chosen.

A cylindrical shape was chosen in order to allow the insertion of an outer mud filtrating membrane and a protective mechanical grid. The sensor element is surrounded by enough space to promote the flow of water allowing updating salinity measurements. The optical cable is fixed at both ends by mechanically blocking the fiber at the head's support, leaving about $11 \mathrm{~cm}$ of exposed optical fiber. The device allows the adjustment of the tension in the fiber through a spring. Fig. 12 presents a picture of the prototype.

\subsubsection{String dimensioning}

The coil spring used in the device was scaled by imposing a force (compression) of $20 \mathrm{~g}$ over a diameter of $40 \mathrm{~mm}$. The spring, with a free length of $130 \mathrm{~mm}$, was made of stainless steel which has an elasticity modulus of $80 \mathrm{GPa}$ and a wire diameter of $1 \mathrm{~mm}$. The number of active coils necessary can be calculated from the equation (5). So, the number of active coils is 14. Assuming squared and ground ends, the total number of the coils is 16 (equation 8).

\subsubsection{Filtering system}

The selection of the membranes used in this work was done taking into account the environment where they are to be inserted and through well-defined parameters such as its material, thickness and pore size. Three types of membranes were analysed with application in aqueous environments, two with the Nylon and the other with fibreglass mesh. Its specifications are shown in Table 1. 


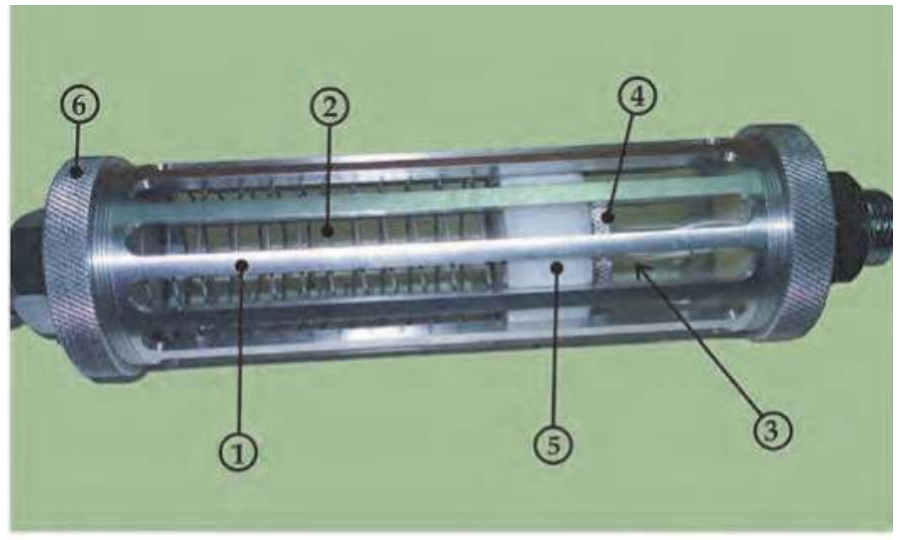

1. Cylinder (membrane support)

2. Spring (control fiber strain)

3. Semi-blocker of optical fibre

\section{Plunger}

5. Ring small bolt

6. Ring bolt-fixing

Fig. 12. Picture of the mechanical device developed to support the element sensor.

\begin{tabular}{|c|c|c|c|c|}
\hline & \multirow{2}{*}{$\begin{array}{c}\text { Mesh } \\
\text { thickness } \\
{[\mu \mathrm{m}]}\end{array}$} & \multirow{2}{*}{$\begin{array}{c}\text { Pore } \\
\text { dimension } \\
{[\mu \mathrm{m}]}\end{array}$} & \multicolumn{2}{|c|}{$\begin{array}{c}\text { Maximum temperature } \\
{[\mathrm{K}]}\end{array}$} \\
\cline { 4 - 5 } & 0.52 & 2.7 & (dry environment) & (wet environment) \\
\hline Fiberglass & 60 & 53 & - & 388 \\
\hline Nylon 53 & 60 & 100 & \multicolumn{2}{|c|}{373} \\
\hline Nylon 100 & 78 & & \multicolumn{2}{|c|}{38} \\
\hline
\end{tabular}

Table 1. Specifications of the analyzed membranes (Cole-Parmer instrument Co).

The analysis to establish the most suitable of these filters was made in situ. Supports were projected based on a net applied surrounding two metallic plates, and over which the membrane to test is fixed (Fig. 13). All metallic parts were designed in aluminium. After placing the membranes in the medium, they were immersed in the Ria de Aveiro, Portugal, where they remained for one month. After this period of time, the membranes were analysed regarding their state of blockage using an optical microscope (Meiji). Inside the vessel, another membrane was placed and a similar analysis was made in order to have an idea of the amount of impurities that have passed the outer filter.

In Fig. 14, it can be observed the state presented by the membranes after the specified period under real conditions. It was found that the fiber glass membrane did not supported the conditions of the environment concerned, and may well be conclude that it is not suitable for the application in question. It can also be seen that for the same period, the nylon membrane with pore size of $53 \mu \mathrm{m}$ shows greater accumulation of dirt compared to the nylon membrane pore with a size of $100 \mu \mathrm{m}$, as expected. Fig. 15 shows the microscope images for both nylon membranes. The nylon membrane with larger pore sizes was fully saturated, while the other still had some open pores. However, because of its condition, it appears that the replacement, in real operation, has to be done in less than a month. The nylon membrane with pore size of $53 \mu \mathrm{m}$ placed inside the support was also relatively clean. 


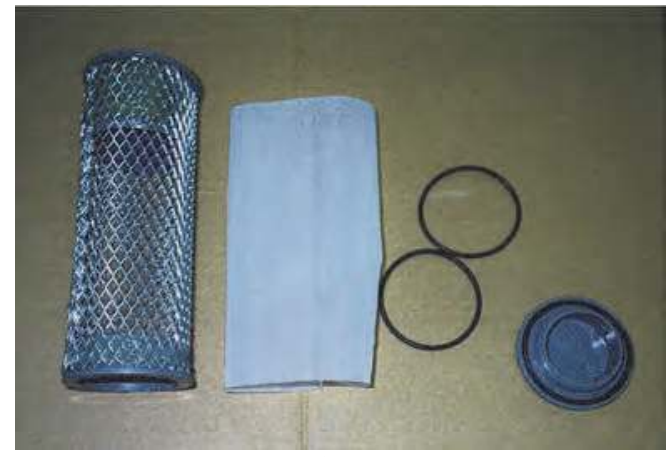

(a)

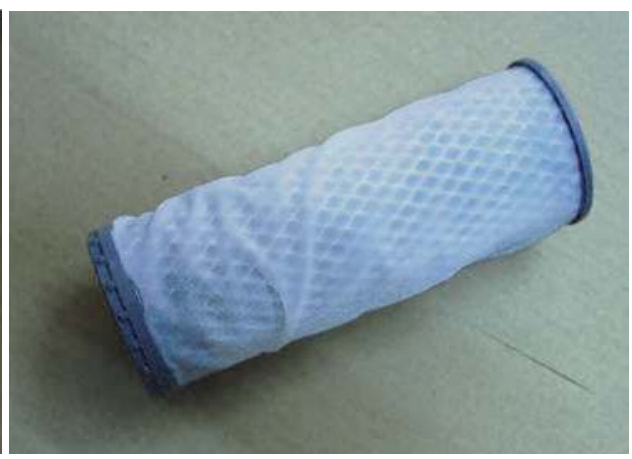

(b)

Fig. 13. (a) Components and (b) membrane mounted on its support for testing.

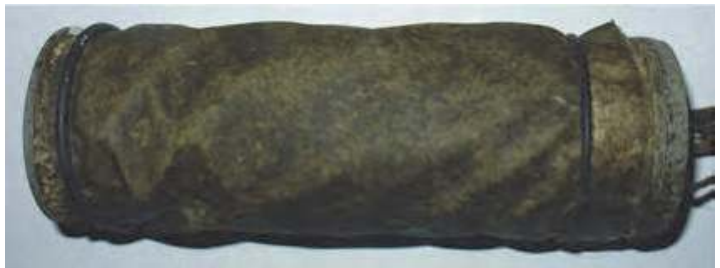

(a)

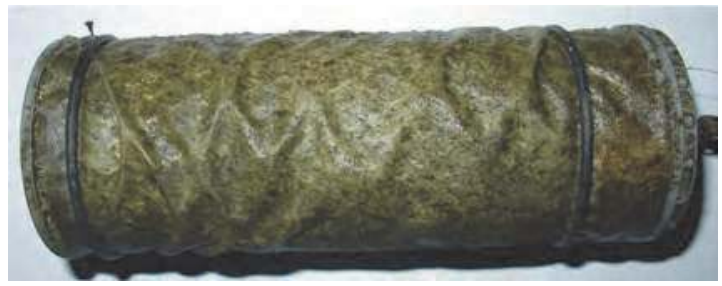

(b)

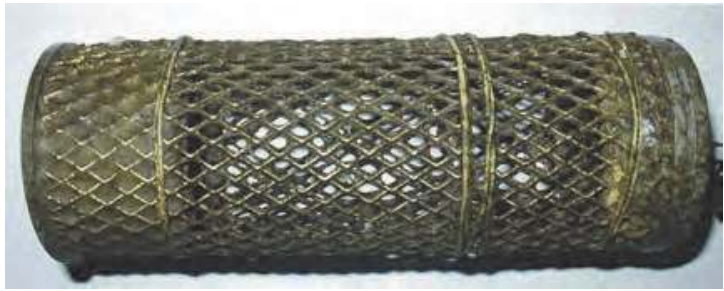

(c)

Fig. 14. Condition of the membranes after immersed in the waters of the Ria de Aveiro, Portugal, for one month: (a) Nylon $(100 \mu \mathrm{m})$, (b) Nylon $(53 \mu \mathrm{m})$ and (c) Fiberglass.

According to the results presented we can conclude that for the application in question nylon fiber with $53 \mu \mathrm{m}$ pore size is the one that best meets the requirements. However, its replacement should occur in relatively short periods (at least every two weeks). 


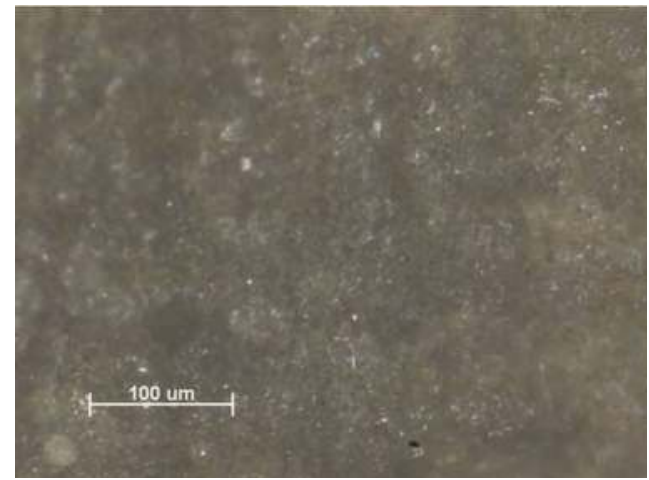

(a)

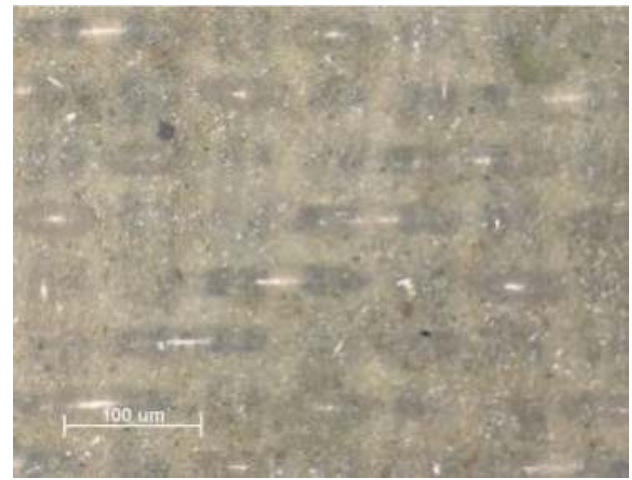

(b)

Fig. 15. Images obtained from optical microscopy of the Nylon membranes, (a) $100 \mu \mathrm{m}$ and (b) $53 \mu \mathrm{m}$, after immersed in the waters of the Ria de Aveiro, Portugal, for one month.

These tests also allowed analysing the performance of an aluminium structure subjected to the aqueous harsh conditions of Ria de Aveiro. In spite of its susceptibility to corrosion, the structure supported relatively well the one month "ordeal". However, the existing screw threads were clearly damaged, showing a tendency to weld the parts in contact.

\section{Sensing head performance}

The performance of the previously defined system is analysed in this section. In order to evaluate the influence of the structure on the measurement of environmental parameters, a comparative study is made when the LPG is fixed with and without the structure. The sensitivity of the sensing head to the refractive index change of the external medium was characterized. The thermal response of the sensing structure was also studied. The LPG used in this work were written by electric arc technique (Rego et al., 2005) in a standard telecommunication fibre (Corning SMF-28), with a period of $\sim 540 \mu \mathrm{m}$ and a length of $\approx 2$ $\mathrm{cm}$. To further enhance its sensitivity for refractive index changes, the fibre diameter was reduced. This was made by chemical attack with aqueous solution of fluoridic acid (HF $40 \%)$. After the etching process, the fibre was thoroughly washed with deionised water, dried, and observed under the microscope. The diameter of the fibre after suffering the chemical etch was $118.8 \mu \mathrm{m} \pm 1 \mu \mathrm{m}$, considering an initial diameter of $125 \mu \mathrm{m}$.

\subsection{Refractive index measurement}

To check the suitability of the system developed for salinity refractive index measurements, the sensitivity of the LPG based sensing head was characterized towards changes in the refractive index of the external medium. For calibration purposes, in both situations the LPGs were immersed in liquids with different refractive indexes at room temperature. The liquid was prepared by using a mixture of water and different ethylene glycol concentrations were used, resulting in samples with refractive indexes in the range [1.33, 1.43]. The refractive index of the different samples was calibrated using an Abbe refractometer. A broadband light source (Photonetics - Fiber White), with emission centred on $1550 \mathrm{~nm}$ illuminated the sensing element. From the transmitted broadband spectrum, the 
refractive index can be measured from the spectral shift of the LPG attenuation band. This shift was monitored using an optical-spectrum analyzer (ANDO AQ 6330). Fig. 16 shows the grating responses to the surrounding solutions with increasing concentration of ethylene glycol, with the device either free (without curvature) or integrated in the structure.

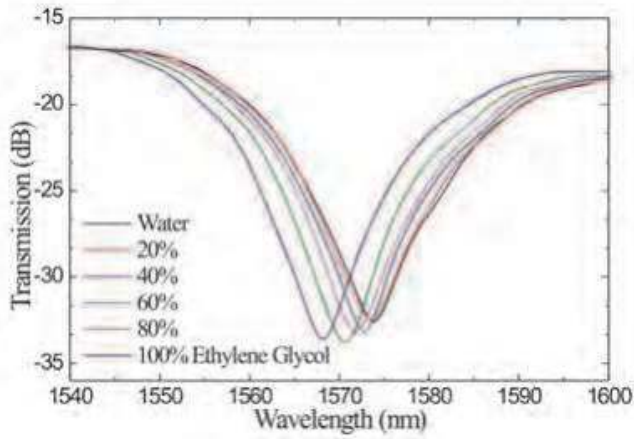

(a)

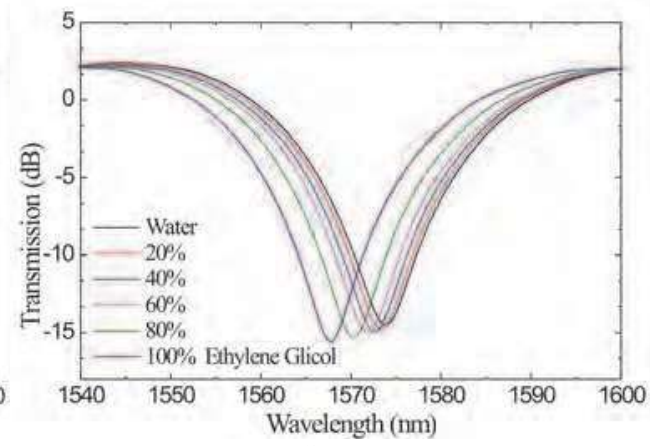

(b)

Fig. 16. Evolution of the transmission spectra of the LPG, (a) free and (b) mounted in the sensing head, as the refractive index is changed by increasing the concentration of ethylene glycol.

It can be seen that the resonance wavelength moves towards the shorter wavelength region as the concentration of ethylene glycol is increased. This can be understood through an increase of the effective refractive index of the cladding, which, according to the resonance condition, leads to lower resonant wavelengths (Lee et al., 1997). The graph presented in Fig. 17 derives directly from the analysis of the central peak of each attenuation band presented in Fig. 15. The shift is determined with reference to the spectral position considering air as the surrounding medium.

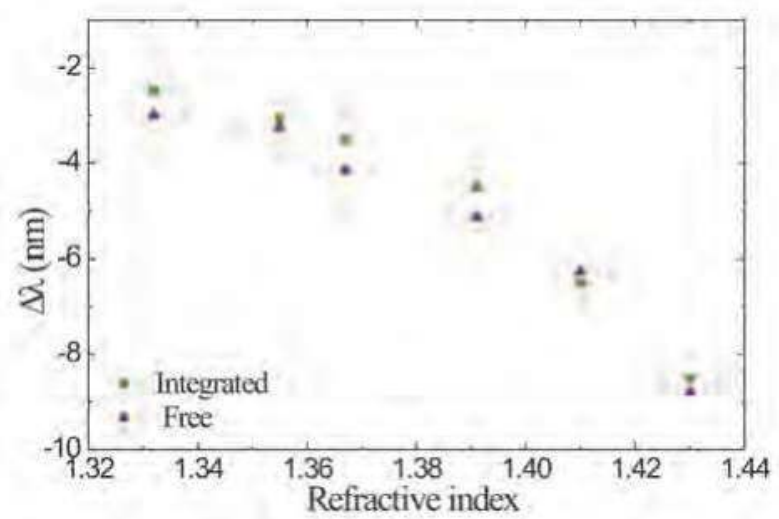

Fig. 17. Resonance wavelength shift of the LPG measured for different refractive indexes of the external medium in the situations where the device is free and integrated in the structure. 
It can be seen that for both situations there is a similar response of the grating to refractive index changes. The slope of the obtained calibration curves gives an idea about the sensitivity of the refractometer. For a refractive index around 1.40, the sensitivity of the LPG is $\approx-67 \mathrm{~nm} /$ RIU and $-57 \mathrm{~nm} /$ RIU for the cases where the device is mounted and free, respectively (RIU: refractive index unit). This difference is an indication that the pre-strain of the LPG in the structure induces a small enhancement of its refractive index sensitivity. As expected, it was also observed that the sensitivity increases substantially when the refractive index of the external medium is close to the cladding refractive index.

\subsection{Salinity measurement}

In order to analyse the influence of the salt concentration, the spectral responses of the LPG mounted in the structure was measured when it was immersed in water with different salt concentrations, resulting into salinity levels between $1 \%$ and $19 \%$. The spectral response evolution of the LPG when mounted in the structure for different salinity levels is shown in Fig. 18, for salt water solutions with different weight concentrations ranging from $0 \%$ to $19 \%$. It can be observed that the resonance wavelength moves towards the shorter wavelength region (shift of $\approx 2 \mathrm{~nm}$ for the salt concentration range tested). This is due to the fact that, with increasing concentration of salt, there is an increase in the refractive index of the solution. Also, it turns out that the concentration of the salt solution does not substantially affect the amplitude of the LPG loss band (variation less than $0.07 \mathrm{~dB}$ ). The resonance wavelength shift with the salt concentration, from where it can be derived a sensitivity of $\approx-0.10 \mathrm{~nm} / \%$ Salt.

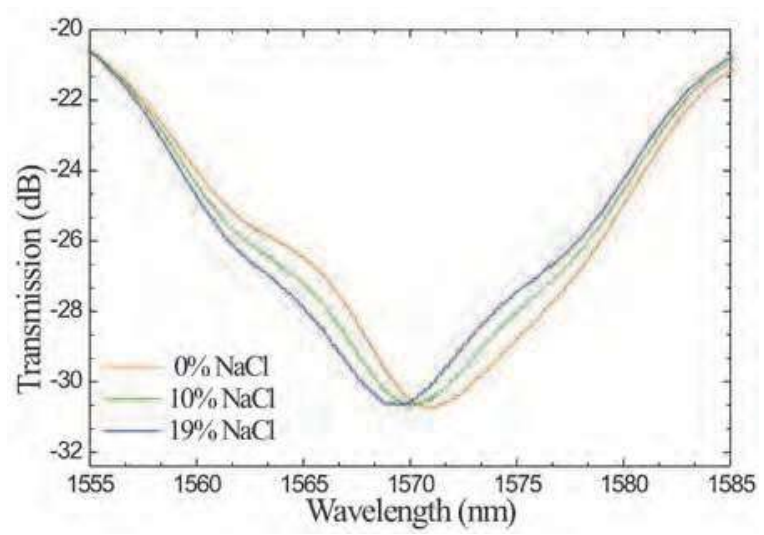

Fig. 18. Transmission spectra of the integrated LPG sensing head in an aqueous solution containing increasing concentration of salt.

\subsection{Temperature measurement}

To study the influence of the temperature on the developed structure, the system was placed into a thermostatic oven. The temperature was changed in increments of $5^{\circ} \mathrm{C}$ from $25^{\circ}$ $\mathrm{C}$ to $100^{\circ} \mathrm{C}$, in the air. The results are shown in Fig. 19. It is evident that the wavelength resonance shift is approximately linear up to $\approx 40^{\circ} \mathrm{C}$, with a slope of $\approx 46 \mathrm{pm} /{ }^{\circ} \mathrm{C}$. At this 
temperature there is a change, certainly due to some thermal induced mechanical adjustment in the sensing structure. From $45^{\circ} \mathrm{C}$ to $100^{\circ} \mathrm{C}$, the behaviour keeps approximately linear but now with a slope of $\approx 60 \mathrm{pm} /{ }^{\circ} \mathrm{C}$. Anyway, the sensing head was designed for application in aqueous environments where the expected temperature range is between $0{ }^{\circ} \mathrm{C}$ and $30^{\circ} \mathrm{C}$. For this range, the thermal sensing behaviour is fairly linear and reversible, with a coefficient $\left(\approx 40 \mathrm{pm} /{ }^{\circ} \mathrm{C}\right)$, which is different from the one found in a free LPG written in standard SMF28 fibre $\left(\approx 70 \mathrm{pm} /{ }^{\circ} \mathrm{C}\right)$. This difference is certainly due to the fact that the LPG in the sensing head is under tension, and therefore the thermal induced shift of the LPG resonance is also affected by the thermal behaviour of the sensing head mechanical structure.

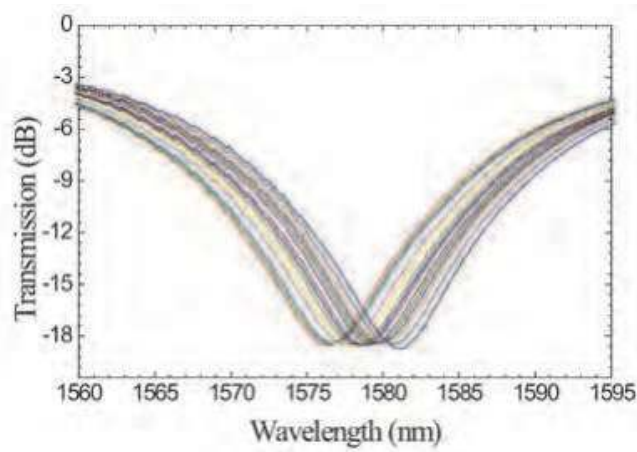

(a)

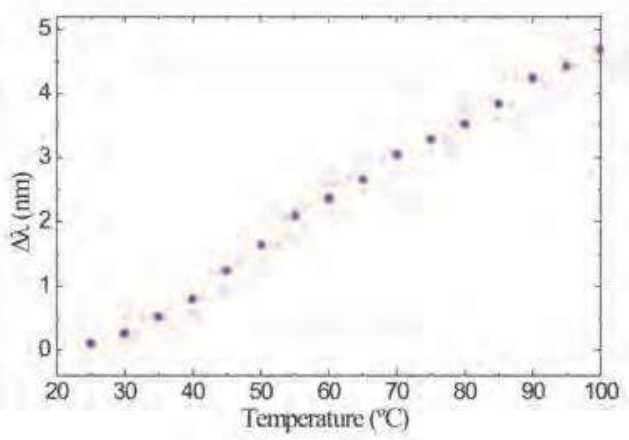

(b)

Fig. 19. Variation of the LPG resonance with temperature for the integrated sensing head.

From the results obtained it is clear that the problem of discriminating temperature induced shifts from refractive index induced wavelength shifts must be addressed in a real operational system. Nevertheless, many solutions are described in the literature that can be directly applied in our sensing head. The inscription of a single FBG in the vicinity of the LPG, for instance, would allow to univocally retrieving the temperature. Other option, was recently described [Jesus et al., 2009], using a pair of FBG that were inscribed after the LPG. However, their spectral position was chosen in such a way that each FBG was in opposite sides of the LPG resonance peak. Therefore, in this situation, the ratio of the reflected intensities of each FBG was modulated by the LPG shift being responsive to temperature and refractive index. On the other hand, the FBG wavelength was responsive only to temperature. The authors demonstrated that such a system can be operated in reflection, doubling the sensitivity to refractive index and enabling simultaneous determination of temperature and refractive index. From the practical point of view, such a system could be easily adapted into our robust sensing head protection system.

\section{Conclusion}

The preservation of species found in estuaries and coastal environments is achieved through constant monitoring of water quality, particularly parameters such as temperature and salinity. In the field of environmental monitoring, optical fibre refractometers (OFR) offer 
many advantages over conventional methods, such as immunity to electromagnetic interference, low weight, small dimensions and low insertion losses. Because of these favourable characteristics, the interest in developing fibre optic as salinity sensors has increased in the past few years, some of them based on refractive index measurements. In particular, long-period fibre gratings (LPGs) have attracted a lot of attention because of their high sensitivity to the refractive index of the surrounding environment.

This chapter focused the possible application of long-period gratings technology in environmental monitoring particularly in the measurement of surrounding refractive index or salinity. The features of this grating type were presented and it was proposed and experimentally demonstrated a prototype of a salinity sensor based on a LPG. The sensor element was written on a standard single-mode telecommunication optical fibre (Corning SMF-28) by electric arc discharge. By reducing the cladding diameter of the sensor, an improved measured resolution of $5.75 \times 10^{-5}$ RIU over a measureable refractive index range from 1.33 to 1.43 (at the wavelength of $1550 \mathrm{~nm}$ ) can be achieved, for a cladding diameter of $81 \mu \mathrm{m}$. It was analyzed the response of different gratings to refractive index changes with and without the structure developed, as well as its thermal response. Some important issues related with the LPG implementation in real conditions were presented, regarding the mechanical requirements and a filtration system of a housing support. The proposed robust sensing head has a filtration system for organic materials using the process of membrane separation. The developed system is easy to implement and allows the replacement of the filter without interfering with the rest of the process. Its performance analysis allowed to further underline the engineering aspects that must be taken in consideration when projecting such a system.

\section{Acknowledgment}

The authors gratefully acknowledge José Luis Santos and Orlando Frazão for their advice and crucial contribution. A special thanks to João Martins that designed the mechanical project for the sensing head. The acknowledgments also go to Pedro Corte-Real for his contribution with the schematics presented in this chapter. Finally, we would like to thank to José Manuel Rebordão for his encouragement.

\section{References}

Akiyama, M.; Nishide, K.; Shima, K., Wada, A. \& Yamauchi, R. (1998). A novel long-period fibre grating using periodically releases residual stress of pure-silica core fiber. Optical Fiber Communication Conference and Exhibit, Technical Digest, pp. 276277, ISBN 1-55752-521-8, San Jose, CA , USA, pp. 22-27.

Allsop, T.; Floreani, F.; Jedrzejewski, K.; Marques, P. \& Romero, R. (2006). Spectral characteristics of tapered LPG device as a sensing element for Refractive Index and Temperature. Journal of Lightwave Technology., Vol. 24, No.2, pp. 870-878.

Bhatia, V. \& Vengsarkar, A. (1996). Optical fiber long-period grating sensors. Optics Letters, Vol.21, No.9, pp. 692-694.

Bhatia, V. (1999). Applications of long-period gratings to single and multi-parameter sensing. Optics Express, Vol.4, No.11, pp. 457-466. 
Cardenas-Sevilla, G.; Monzon-Hernandez, D.; Torres-Gomez, I. \& Martinez-Rios, A., (2009). Mechanically induced long-period fiber gratings on tapered fibers. Optics Communications, Vol.282, No.15, pp. 2823-2826.

Cole-Parmer catalogue (2005/06) Cole-Parmer Instrument Company, US.

Davis, D.; Gaylord,T.; Glytis, E.; \& Mettler, S. (1998).Long period fibre grating fabrication with focused $\mathrm{CO}_{2}$ laser pulses. Electronics Letter, Vol.34, No.3, pp. 302-303, ISSN 0013-5194.

Ding, J.; Zhang, A.; Shao, L.; Yan J. \& Sailing H. (2005). Fiber-taper seeded long-period grating air as a highly sensitive refractive-index sensor. IEEE Photonics Technology Letters, Vol.17, No.6, pp. 1247-1249.

Dong, X., L. Su, P. Shum, Y. Chung, \& C. C. Chan (2005). Photonic-Crystal-Fiber-Based Mach-Zehnder Interferometer using Long-Period Gratings. Optics Communications, Vol.258, No.159, pp.1379-1383.

Dong, X.; Feng, S. ; Xu, O.; Lu, S.; \& Pei, L. (2009). Add/drop channel filter based on two parallel long-period fiber gratings coupler. Optik, Vol. 120, pp. 855-859.

Erdogan, T. (1997a). Fiber grating spectra. Journal of Lightwave Technology. Vol.15, No.8, pp. 1277-1294.

Erdogan, T. (1997b). Cladding-mode resonances in short- and long-period fiber grating filters. Journal of the Optical Society of America A, Vol.14, pp.1760-1773.

Falate, R.; Frazão, O.; Rego, G.; Fabris, J. \& Santos, J. (2006). Refractometric sensor based on a phase-shifted long-period fiber grating. Applied Optics, Vol.45, No.21, pp. 50665072.

Falciai, R., Mignani, A., \& Vannini, A. (2001).Long period gratings as solution concentration sensors Sensors and Actuators B: Chemical, Vol.74, No.1-3, 15 pp. 74-77. Proceedings of the 5th European Conference on Optical Chemical Sensors and Biosensors.

Fujimaki, M.; Nishihara, Y., Ohki, Y.; Brebner, J. \& Roorda S. (2000). Ion-implantationinduced densification in silica-based glass for fabrication of optical fiber gratings. Journal of Applied Physics, Vol.88, No.10, pp. 5534-5537, ISSN 0021-8979.

Frazão, O.; Falate, R.; Fabris, J.; Santos, J. \& Ferreira, L. (2006). Optical inclinometer based on a single long-period fiber grating combined with a fused taper. Optics Letters, Vol. 31, No. 20, pp 2960-2962.

Gwandu B., Shu X., Allsop T., Zhang W. \& Zhang L. (1999). Simultaneous refractive index and temperature measurement using cascaded long period grating in double cladding fibre. Electronics Letters, Vol.35, No.14, pp. 695 - 696, ISSN 0013-5194.

Han, Y.; Lee, B.; Han, W.; Paek, U. \& Chung, Y (2001). Fiber optic sensing applicatios of a pair of long period fibre gratings. Measurement Science and Technology, Vol.12, 778781.

Hill, O.; Malo, B.; Vineberg, K., Bilodeau, F.; Johnson, D. (1990). Efficient mode conversion in telecommunication fiber using externally written gratings. Electronics Letters, Vol. 26, pp. 1270-1272.

Humbert G. \& A. Malki (2002). Characterizations at very high temperature of electric arcinduced long-period fiber gratings. Optics Communications, Vol.208, No.4-6, pp. 329335 . 
Humbert, G. \& Malki A. (2003). High performance bandpass filters based on electric arcinduced п-shifted long-period fibre gratings. Optics Express, Vol.39, No.21, pp. 1506-1505, ISSN 0013-5194.

James, S.; Rees, N.; Ashwell, G. \& Tatam, R. (2002). Optical Fiber long-period Grating with a Langmuir -Blodgett thin film overlays. Optics Letters, Vol.9, pp. 686-688.

James, S. \& Tatam, R. (2003). Optical fibre long-period grating sensors: Characteristics and application. Measurement Science and Technology, Vol. 14, pp. R49-R61.

Jesus, C.; Caldas, P.; Frazão, O.; Santos, J.; Jorge, P. \& Baptista, J. (2009).Simultaneous Measurement of Refractive Index and Temperature Using a Hybrid FBG/LPG Configuration. Fiber Integrated Optics, Vol.28, pp 440-449.

Kashyp, R. (2010). Fiber Bragg Gratings. 2nd ed, Optics and Photonics Series, Academic Press, Boston.

Khaliq, S.; James, S.; \& Tatam R. (2002). Enhanced sensitivity fibre optic long period grating temperature sensor. Measurement Science and Technology, Vol.13, No.5, pp 792-795.

Kim, B. ; Ahn, T. ; Kim, D. ; Lee, B. \& Chung, Y. (2002). Effect of CO2 laser irradiation on the refractive-index change in optical fibers. Applied Optics, Vol.41, No.19, pp. 38093815.

Kondo, Y.; Nouchi, K.; Mitsuyu, T.; Watanabe, M. \& Kazansky, P. (1999). Fabrication of long-period fiber gratings by focused irradiation of infrared femtosecond laser pulses, Optics Letters, Vol. 24, pp. 646-648.

Lee, B.; Liu, Y.; Lee, S.; Choi, S. \& Jang, J. (1997). Displacement of the resonant peaks of a long-period fiber grating induced by a change of ambient refractive index. Optics Letters, Vol.22, pp. 1769-1771.

Lee, B.; Nishii, J. (1998). Self-interference of long period fibre gratings and its application as temperature sensor. Electronics Letters, Vol.34, pp. 2059-2060.

Lemaire, P.; Atkins, R.; Mizrahi, V. \& Reed W. (1993). High pressure H2 loading as a technique for achieving ultrahigh UV photosensitivity and thermal sensitivity in $\mathrm{GeO}_{2}$ doped optical fibres. Optics Express, Vol.29, pp. 1191.

Liu, Y.; Tu, W.; Yang, D. \& Wang T. (2011), Fabrication of long-period fiber gratings by $\mathrm{CO}_{2}$ laser in fiber under tension. Journal of Shanghai University, (English Edition), Vol.15, No.1, pp. 1-6.

Murphy, R.; James, S. \& Tatam, R. (2007). Multiplexing of Fiber-Optic Long-Period Grating-Based Interferometric Sensors. Journal of Lightwave Technology, Vol.25, No.3, pp. 825-829.

Mishra, V.; Singh, N.; Jain, S.; Kaur, P. \& Luthra, R. (2005). Refractive index and concentration sensing of solutions using mechanically induced long period grating pair. Optical Engineering, Vol. 44, pp. 094402-1-094402-4.

Nikogosyan D. (2006). Long-period Gratings in a standard telecom fibre fabricated by highintensity fentosecond UV and near-UV laser pulses. Measurement Science and Technology. Vol.17, pp. 960-967.

Patrick, H.; Chang, C. \& Vohra, S. (1998a). Long period fiber gratings for structural bending sensing. Electronics Letters, Vol.34, pp. 1773 - 1775. 
Patrick, H., Kersey, A. \& Bucholtz, F. (1998b). Analysis of the response of long-period fiber gratings to external index of refraction. Journal of Lightwave Technology. Vol.16, pp. 1606-1612.

Queiroz S.; Collins, C. \&; Jardim, I. (2001) .Métodos de extracção e/ou concentração de compostos encontrados em fluidos biológicos para posterior determinação cromatográfica. Quim. Nova, 24, No 1, 68-76.

Rego G., O. Okhotnikov, E. Dianov, \& V. Sulimov, (2001). High-temperature stability of long period fiber gratings produced by using an electric-arc. Journal of Lightwave Technology, Vol.19, pp. 1574-1579.

Rego G., Fernandes,J.; Santos, J.; Salgado, H. \& Marques, P. (2003). New technique to mechanically induce long-period fibre gratings. Optics Communications, Vol. 220, No. 1-3, pp. 111-118.

Rego, G. ; Marques, P. ; Santos, J. \& Salgado, H. (2005). Arc-induced Long-period Gratings. Fiber and Integrated Optics, Vol.24 (3-4), pp. 245-259.

Rego, G.; Santos, J.; \& Salgado, H. (2006). Refractive index measurement with long-period gratings arc-induced in pure-silica-core fibres. Optics Communications, Vol.259, pp. 598-602.

Shifler D. (2005). Understanding material interactions in marine environments to promote extended structural life. Corrosion Science, Vol.47, pp. 2335-2352.

Shigley J. (1986). Mechanical Engineering Design - First Metric Edition, McGraw-Hill S Book Company, ISBN 0-07-056898-7.

Silva, C.; Coelho, J.; Caldas, P. ; Frazão, O.; Jorge, P.; Santos, J. (2010). Optical fibre sensing system based on long-period gratings for remote refractive index measurement in aqueous environments. Fiber and Integrated Optics, Vol.29, No.3, 160-169.

Swart P. L. (2004). A single fiber Michelson interferometric sensor. Proceedings of the 16 th International Conference on Optical Fiber Sensors- Japan, pp. 602-605.

Vengsarkar A.; Lemaire, P.; Judkins, J.; Bhatia, V. \& Sipe, J.(1996a). Long-Period FiberGrating-Based Gain Equalizers. Optics Letters, Vol. 21, pp. 335-338.

Vengsarkar A., Pedrazzani, J.; Judkins J. B.; Lemaire, P. \& Bhatia, V. (1996b). Long-period fiber gratings as band-rejection filters. Journal of Lightwave Technology, Vol.14, No.1, pp. 58-65.

Yokouchi T., Suzaki,Y.; Nakagawa, K.; Yamauchi, M. \& Kimura (2005). Thermal tuning of mechanically induced long-period fiber grating. Applied Optics, Vol.44, No.24, pp. 5024-5028.

Yu, X.; Shum, P. \& Dong, X. (2006). Photonic-crystal-fiber-based-Mach-Zehnder interferometer using long-period gratings. Microwave and optical technology letters, Vol.48, No.7, pp. 1379-1383.

Zhang, A.; Shao, L.; Ding, J. \& He, S. (2005). Sandwiched long-period gratings for simultaneous measurement of refractive index and temperature. IEEE Photonics Technology Letters, Vol.7, No.11, pp. 2397-2399, ISSN 1041-1135.

Zanlorensi R. V., Costa, R. C. Kamikawachi, M. Muller and J. L. Fabris, (2009). Thermal characteristics of long-period gratings $266 \mathrm{~nm}$ UV-point-by-point induced. Optics Communications, Vol.282, No.5, pp 816-823. 
Zhao Y., Y. Liao, B. Zhang, (2003). Monitoring Technology of Salinity in Water With Optical Fiber Sensor. Journal of Lightwave Technology, Vol.21, No.5, pp. 1334-1338.

Zhu Y., J. H. Chong, M. K. Rao, H. Haryono, A. Yohana, Ping Shum, Lu, C. (2003).A longperiod grating refractometer: measurements of refractive index sensitivity. Proceedings SBMOIIEEE MTT-S IMOC, pp. 901-904. 


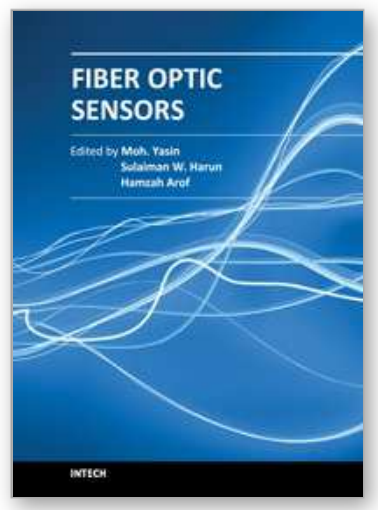

\author{
Fiber Optic Sensors \\ Edited by Dr Moh. Yasin
}

ISBN 978-953-307-922-6

Hard cover, 518 pages

Publisher InTech

Published online 22, February, 2012

Published in print edition February, 2012

This book presents a comprehensive account of recent advances and researches in fiber optic sensor technology. It consists of 21 chapters encompassing the recent progress in the subject, basic principles of various sensor types, their applications in structural health monitoring and the measurement of various physical, chemical and biological parameters. It also highlights the development of fiber optic sensors, their applications by providing various new methods for sensing and systems, and describing recent developments in fiber Bragg grating, tapered optical fiber, polymer optical fiber, long period fiber grating, reflectometry and interefometry based sensors. Edited by three scientists with a wide knowledge of the field and the community, the book brings together leading academics and practitioners in a comprehensive and incisive treatment of the subject. This is an essential reference for researchers working and teaching in optical fiber sensor technology, and for industrial users who need to be aware of current developments and new areas in optical fiber sensor devices.

\title{
How to reference
}

In order to correctly reference this scholarly work, feel free to copy and paste the following:

Catarina Silva, João M. P. Coelho, Paulo Caldas and Pedro Jorge (2012). Fibre Sensing System Based on Long-Period Gratings for Monitoring Aqueous Environments, Fiber Optic Sensors, Dr Moh. Yasin (Ed.), ISBN: 978-953-307-922-6, InTech, Available from: http://www.intechopen.com/books/fiber-optic-sensors/fibresensing-system-based-on-long-period-gratings-for-monitoring-aqueous-environments

\section{INTECH}

open science | open minds

\section{InTech Europe}

University Campus STeP Ri

Slavka Krautzeka 83/A

51000 Rijeka, Croatia

Phone: +385 (51) 770447

Fax: +385 (51) 686166

www.intechopen.com

\section{InTech China}

Unit 405, Office Block, Hotel Equatorial Shanghai

No.65, Yan An Road (West), Shanghai, 200040, China

中国上海市延安西路 65 号上海国际贵都大饭店办公楼 405 单元

Phone: +86-21-62489820

Fax: $+86-21-62489821$ 
(C) 2012 The Author(s). Licensee IntechOpen. This is an open access article distributed under the terms of the Creative Commons Attribution 3.0 License, which permits unrestricted use, distribution, and reproduction in any medium, provided the original work is properly cited. 